\title{
Identification of Nanosized Defects Using Tribospectroscopy. Modeling by Movable Cellular Automaton Method
}

\author{
Galina M. Eremina ${ }^{1,2, a)}$, Alexey Yu. Smolin ${ }^{1,2, b)}$, Valery V. Sergeev ${ }^{2, c)}$, \\ and Sergey G. Psakhie ${ }^{1,3, \mathrm{~d})}$ \\ ${ }^{1}$ Institute of Strength Physics and Materials Science SB RAS, Tomsk, 634055, Russia \\ ${ }^{2}$ National Research Tomsk State University, Tomsk, 634050, Russia \\ ${ }^{3}$ Institute of High Technology Physics, National Research Tomsk Polytechnic University, Tomsk, 634050, Russia \\ a) Corresponding author: anikeeva@ispms.tsc.ru \\ b) asmolin@ispms.tsc.ru \\ ${ }^{c)}$ svalera@ispms.tsc.ru \\ ${ }^{\mathrm{d})} \mathrm{sp} @$ ispms.tsc.ru
}

\begin{abstract}
The work describes the application of the movable cellular automaton method for the simulation of typical situations of nano-scale crack identification in ceramic coatings by the spectral analysis of friction force continuously registered using a small counter-body moving over the coating surface. It is shown that the Fourier spectra of the friction force have peaks that correspond to the distance between the nanocracks oriented perpendicular and at some angle to the tested surface. The obtained results allow to conclude that the appearance of cracks on the Fourier spectra of friction force depends on their orientation. Thus, the distance between cracks, their orientation and sizes can be identified by the method of tribospectroscopy.
\end{abstract}

Keywords: non-destructive testing, tribospectroscopy, coating, nanocracks, modeling, movable cellular automaton method

\section{INTRODUCTION}

The nanostructured coatings can significantly improve the operational characteristics of machine parts. In this case, the mechanical properties of these coatings strongly depend on the presence of defects and damages of nanosize scale. In this regard, it is urgent to develop methods for diagnosing such defects [1]. One of the methods of the nondestructive testing of coatings which allows to assess structural defects in thin surface layers of solids could be based on the approach that implements friction coefficient as measuring parameter of the system response [2,3], namely tribospectroscopy. This method is based on the analysis of sliding friction when a small rider (indenter) is moving along the surface of the coating.

The possibility of tribospectroscopy application for the analysis of defects in the surface layers of solids with sufficient detail can be studied by numerical simulations. Similar studies were previously conducted for both twodimensional and three-dimensional problems (for example, using a high-strength ceramic coating). Nanocracks in the form of transparent cavities oriented in the direction transverse to the movement of the indenter (i.e. perpendicular to the plane of two-dimensional problem) are extended along this direction [1-4].

\section{STATEMENT OF PROBLEM AND RESULTS}

Movable cellular automaton method which is a new efficient numerical method in particle mechanics is used herein for modeling motion of the small counter-body over the coating surface. Within the frame of movable cellular automaton, it is assumed that any material is composed of a certain amount of elementary objects (automata) that

(C) 2014 AIP Publishing LLC 978-0-7354-1260-6/\$30.00 
interact among each other and can move from one place to another, thereby simulating a real deformation process [5, 6]. The automaton motion is governed by the Newton-Euler equations. The forces acting on automata are calculated using the deformation parameters, i.e. relative overlap, tangential displacement and rotation, and conventional elastic constants, i.e. shear and bulk moduli. A distinguishing feature of the method is calculating the forces acting on the automata within the framework of multi-particle interaction [7], which provides for an isotropic behavior of the simulated medium. Moreover, stress tensor components can be calculated for the automaton taking into account all the forces acting on it, which enables realization of various models of elastic and plastic behavior developed in the frame of continuum mechanics.

A pair of elements can be considered as a virtual bistable cellular automaton (bound and unbound states), which enables the simulation of fracture by the movable cellular automaton. Switching over the pair of automata to the unbound state would result in a changeover of the forces acting on the elements; in particular, they will not resist moving away from one another. Removing automata from initial dense packing allows explicit analysis of voids or pores in material.

The coating of $\mathrm{ZrO}_{2}$ ceramics on a steel substrate has been modeled (Fig. 1(a)). The thickness of the coating $H$ was $60 \mathrm{~nm}$. The model sample length $L=500 \mathrm{~nm}$, width $M=125 \mathrm{~nm}$, the size of the automata $d=3 \mathrm{~nm}$. Ceramic counter-body had a conical shape with the base diameter of $60 \mathrm{~nm}$.

Motion of the counter-body was simulated by setting the constant velocity $V=5 \mathrm{~m} / \mathrm{s}$ in the direction of $Y$-axis for automata of the upper layer of the counter-body (Fig. 1(a)). The lower surface of the sample was fixed and its lateral surfaces were free. When the counter-body was moving the resistance force of its movement on the surface was recorded and correlated with the friction force $F$ varying in time $t$. The Fourier transform was made for the registered friction force $F(t)$ and the corresponding spectra were analyzed [4].

The damage of the coating was simulated by specifying the extended coating discontinuities, nanocracks. These nanocracks were located periodically at the predetermined distance from each other. The distance between the cracks and their orientation relatively to the test surface was varied. The ability of determining the size and spatial arrangement of these defects according to the spectra of registered $F(t)$ was analyzed.

In [4] it was shown that the peak characterizing the repeating period of the nanocracks in two-dimensional model specimen was influenced by the surface bending when the counter-body was passing above the defect. The more the crack length was, the bigger amplitude the corresponding peak had. The deeper was the defect, the lower was the amplitude. The data was obtained using the specimen with plain geometry, which supposes the cracks to be transparent in the direction perpendicular to the specimen plain. The possibility of peaks identification for threedimensional statement characterizing the parameters of model specimens was investigated elsewhere [8, 9]. The registration of the peaks involved the coating bending above the cracks, the bending being induced by the indenter, similarly to the two-dimensional statement of the problem.

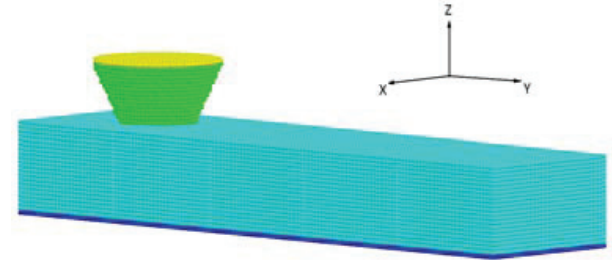

(a)

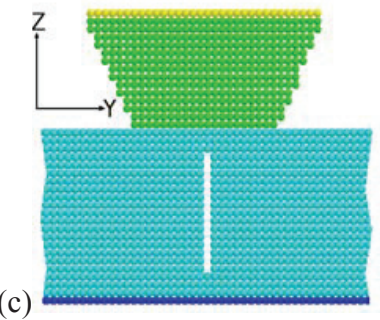

(b)

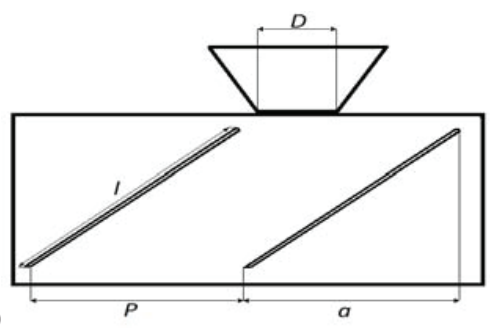

(d)

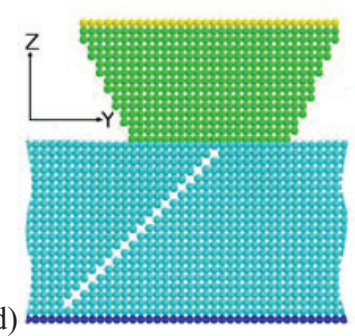

FIGURE 1. Modeled system: (a) general view, (b) the schematic representation of modeled system with nanocracks, (c) perpendicular crack and (d) inclined crack 


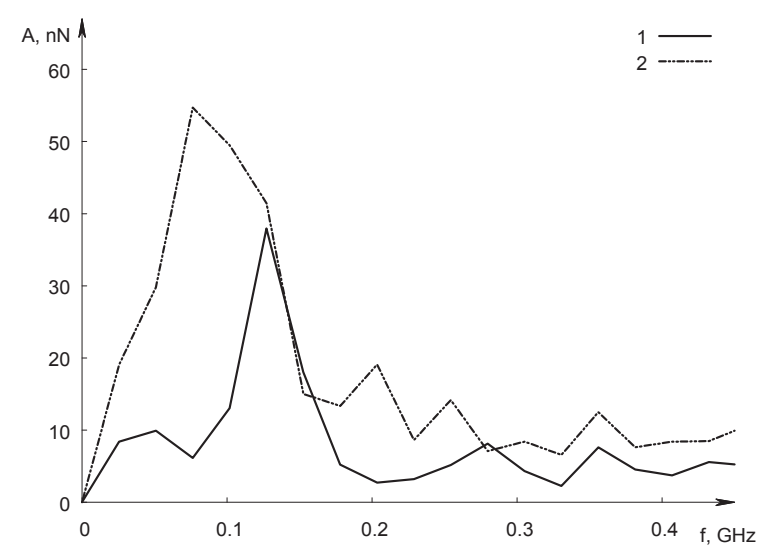

FIGURE 2. Fourier spectra of the force $F(t)$ acting on the counter-body moving along specimen with perpendicular nanocracks spaced at a) $96 \mathrm{~nm}$ and 2) $51 \mathrm{~nm}$ from each other

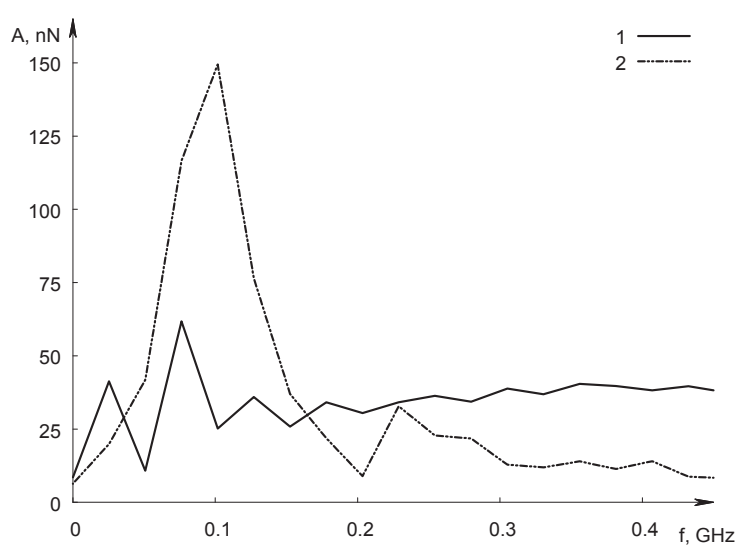

FIGURE 3. Fourier spectra of the force $F(t)$ acting on the counter-body moving along specimen with inclined nanocracks spaced at 1) $96 \mathrm{~nm}$ and 2) $78 \mathrm{~nm}$ from each other

This paper studies the three-dimensional specimens with nanocracks oriented perpendicularly to the test surface and at the inclination of $45^{\circ}$ (Fig. 1), the distance between the cracks being varied.

Figure 2 shows the Fourier spectra for the case of perpendicular nanocracks, the distance between the cracks being varied. The frequency characterizing the distance between failures is determined by the formula proposed in [9]:

$$
f=V /(P-D),
$$

where $V$ is the velocity of the counter-body, $P$ is the distance between cracks, $D$ is the diameter of the indenter (Fig. 1(b)). For the case of perpendicular failures spaced at $96 \mathrm{~nm}$ this frequency is equal to $0.125 \mathrm{GHz}$, and for the failures spaced at $51 \mathrm{~nm}$ from each other, $0.25 \mathrm{GHz}$. These frequencies are presented in the plots of Fig. 2. Moreover, on the curve 2 in Fig. 2 there is the peak of $0.087 \mathrm{GHz}$ characterizing the size of nanocrack, which frequency was defined by the following formula:

$$
f=V / 1,
$$

where $l$ is the size of nanocrack (Fig. 1(b)).

The Fourier spectrum for the inclined nanocracks (Fig. 3(d)) have peaks characterizing the distance between the nanodamages calculated by the formula (1). For the specimen with the distance between the nanocracks equal to $96 \mathrm{~nm}$ this frequency is $0.128 \mathrm{GHz}$, and for the specimen with distance between cracks $78 \mathrm{~nm}$ it is $0.277 \mathrm{GHz}$.

On the Fourier spectrum for inclined cracks the frequency of $0.089 \mathrm{GHz}$ occurs that is characterized by the following relation:

$$
f=V / a,
$$

where $a$ is the projection of the nanocrack to the testing surface.

Thus, the peaks presented in the Fourier spectra both for perpendicular and inclined failures in relation to the surface allow determining the distance between them and their dimensions. These calculations prove the assumption that not only the bend of coating above the crack, but also the full path of the elastic waves are important for the damage identification by the tribospectroscopy method [10].

\section{SUMMARY}

On the basis of numerical simulation by the method of movable cellular automaton it is shown that the Fourier spectrum of the friction force affecting the counter-body while it moves along the coating with periodically arranged nanocracks contains the peak corresponding to the distance between cracks. For the inclined cracks the Fourier spectrum contains the frequency peak characterizing the projection of the crack, which allows the estimation of the crack size. The crack orientation influences the different appearance of these cracks on the Fourier spectra of the 
friction force. This allows claiming that the distance between the cracks and their orientation can be identified by tribospectroscopy.

\section{ACKNOWLEDGMENTS}

The investigation was carried out with the partial financial support of the Project No. III.23.2.4 of the Basic Research Program of State Academies of Sciences for 2013-2016.

\section{REFERENCES}

1. V. P. Kuznetsov, I. Yu. Smolin, A. I. Dmitriev, D. A. Konovalov, A. V. Makarov, A. E. Kiryakov, and A. S. Yurovskikh, Phys. Mesomech. 16(1), 62 (2013).

2. V. L. Popov, J. Starcevic, and A. E. Filippov, Phys. Rev. E 75(6), 066104-1 (2007).

3. S. G. Psakhie, V. L. Popov, E. V. Shilko, A. Yu. Smolin, and A. I. Dmitriev, Phys. Mesomech. 12(5-6), 221 (2009).

4. S. G. Psakhie, E. V. Shilko, V. L. Popov, J. Starcevic, J. Thaten, and S. V. Astafurov, Russ. Phys. J. 52(4), 380 (2009).

5. S. Psakhie, E. Shilko, A. Smolin, S. Astafurov, and V. Ovcharenko, Fract. Int. Struct. 24, 26 (2013).

6. S. G. Psakhie, E. V. Shilko, A. Yu. Smolin, A. V. Dimaki, A. I. Dmitriev, Ig. S. Konovalenko, S. V. Astafurov, and S. Zavshek, Phys. Mesomech. 14(5-6), 224 (2011).

7. S. G. Psakhie, A. Yu. Smolin, E. V. Shilko, G. M. Anikeeva, Yu. S. Pogozhev, M. I. Petrzhik, and E. A. Levashov, Comp. Mater. Sci. 76, 89 (2013).

8. V. V. Sergeev, A. Yu. Smolin, S. A. Dobrynin., S. Yu. Korostelev, and S. G. Psakh'e, J. Math. Mech. 4(12), $116(2010)$.

9. A. Yu. Smolin, G. M. Eremina, V. V. Sergeev, E. V. Shilko, and S. G. Psakhie, Phys. Mesomech. 17(4), (2014) (in press).

10. S. G. Psakhie, K. P. Zolnikov., A. I. Dmitriev, A. Yu. Smolin, and E. V. Shilko, Phys. Mesomech. 17(1), 15 (2014). 